\title{
A Case of Secondary Moyamoya Associated with Hyperhomocysteinemia
}

\author{
Authors \\ Azma Naaz Mohammed ${ }^{1 *}$, Narayana Geetha T.L ${ }^{2}$, Sundar Raju Y.G ${ }^{3}$ \\ *Corresponding Author
}

Azma Naaz Mohammed

\begin{abstract}
Moyamoya vasculopathy is a progressive vasculopathy leading to stenosis of main intracranial arteries with the formation of collaterals, which gives a puff of smoke appearance. If it is due to associated condition, it is termed as moyamoya syndrome .one of such association is hyperhomocysteinemia, which is a rare association. Very few cases have been reported in India. Here we report a case of 32-year-old female nonhypertensive, non-diabetic, presented with history of recurrent left side hemiplegia with left UMN Facial nerve palsy. Her routine blood investigations were normal except for macrocytic hyperchromicanemia. On MR Angiography, she was diagnosed to have moyamoyavasculopathy. Thrombophilia profile revealed hyperhomocysteinemia, Which usually present as ischemic stroke, but in our scenario patient presented with moyamoya syndrome, along with intracranial hemorrhage due to fragility of collaterals. In this case, her hyperhomocysteinemia is attributed to low vitamin $b 12$ and folic acid levels. So simple vitamin b12 and folic acid deficiency can cause hyperhomocysteinemia, which in turn leads to stroke in young .Thus nutritional anemia should be corrected as early as possible so that its deleterious effects are prevented.
\end{abstract}

\section{Introduction}

Moyamoya vasculopathy (MMV) is a chronic, progressive cerebrovascular Disease. It is due to stenosis or occlusion of bilateral terminal internal carotid arteries and proximal branches of anterior cerebral artery and middle cerebral artery with the development of collaterals at the base of the brain, which may give the impression of a puff of smoke. MMV is classified as moyamoya disease, which is primary pathology and moyamoya syndrome, which is due to some other associated conditions. One of such associations is hyperhomocysteinemia. Usually, hyperhomocysteinemia presents with ischemic stroke, but in our case patient presented with moyamoya syndrome, along with intracranial hemorrhage due to fragility of collaterals. Hyperhomocysteinemia can be due to umpteen number of causes here in our case, it is due to low vitamin b 12, and folic acid levels .So simple vitamin b12 and folic acid deficiency can cause hyperhomocysteinemia which in turn leads to stroke in young.

\section{Case Report}

A 32-year-old female presented with a history of sudden onset of weakness of the left upper limb and the lower limb, and deviation of angle of mouth to the right side. It was not associated with headache, vomitings, and seizures. There were no sensory symptoms and bladder disturbances. However, she had history of chronic holocranial headache, which is a dull throbbing type not associated with any aggravating and relieving factors for the past one year. She had a similar episode of left-sided 


\section{JMSCR Vol||07||Issue||11||Page 653-656||November}

hemiplegia six months back, which has been recovered with partial residual deficit. She was not hypertensive and diabetic. Family history was not significant.

On general examination, the patient was conscious, cooperative, and coherent. Pulse was 80 beats/minute and regular. All peripheral pulses were palpable. Her blood pressure was $120 / 70 \mathrm{mmHg}$. Respiratory rate was 18 breaths/minute and regular in rhythm. The temperature was $98.4^{\circ} \mathrm{F}$. There was pallor. No icterus, edema, and cyanosis.

On neurological examination, the Glasgow coma scale was 15/15 (E4V5M6). Cranial nerve examination demonstrated upper motor neuron type of left-sided facial nerve palsy; the rest of the cranial nerves examination was normal.

Motor system examination demonstrated hypertonia, with the muscle power of grade $1 / 5$, and brisk deep tendon reflexes of the left upper limb and lower limb. The sensory and cerebellar examination revealed no abnormalities.

Haematological investigations revealed

Table no: 1

\begin{tabular}{|l|c|}
\hline Haemoglobin & $10.0 \mathrm{~g} / \mathrm{dl}$ \\
\hline Red Blood Cell Count & $3.1 \mathrm{million}$ cells/ $\mu \mathrm{L}$ \\
\hline $\mathrm{MCV}$ & $116 \mathrm{fl}$ \\
\hline $\mathrm{MCH}$ & $33 \mathrm{pg}$ \\
\hline $\mathrm{MCHC}$ & $28 \mathrm{~g} / \mathrm{dl}$ \\
\hline Total Platelet Count & $3.4 \mathrm{lakhs} / \mu \mathrm{L}$ \\
\hline Haematocrit & $6700 / \mu \mathrm{L}$, \\
\hline Total Leukocyte Count & $60 \mathrm{~mm}$ in $1 \mathrm{st}$ hour, \\
\hline $\begin{array}{l}\text { Erythrocyte Sedimentation } \\
\text { Rate (ESR) }\end{array}$ & Macrocytic anemia \\
\hline Peripheral smear & Normal \\
\hline $\begin{array}{l}\text { RFT(renal function } \\
\text { tests ) }\end{array}$ & Normal \\
\hline LFT (liver function tests) & Normal \\
\hline $\begin{array}{l}\text { Urine routine } \\
\text { microscopy }\end{array}$ & $64 \mathrm{micro} \mathrm{mol} / \mathrm{l}$. \\
\hline Vitamin b12 levels & \\
\hline Homocysteine & \\
\hline
\end{tabular}

Imaging: CT scan of brain suggestive of right thalamic bleed with ipsilateral intraventricular extension.MR Angiography showed right thalamic bleed and severe stenosis of bilateral internal carotid arteries with abnormal net-like collaterals in left cerebral parenchyma and deep gray matter regions consistent with moyamoya disease.

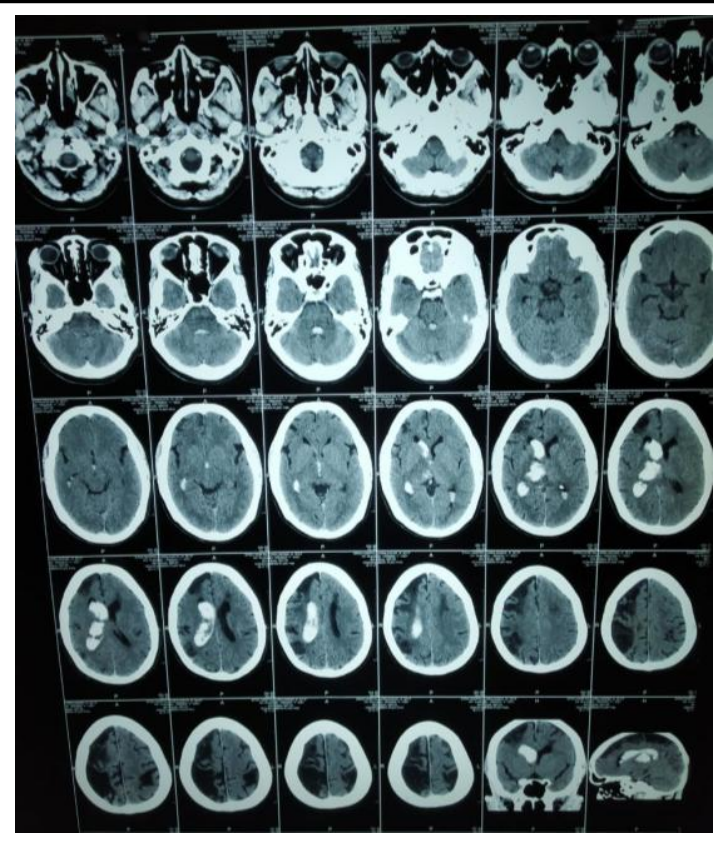

Figure-1 CT Brain Plain

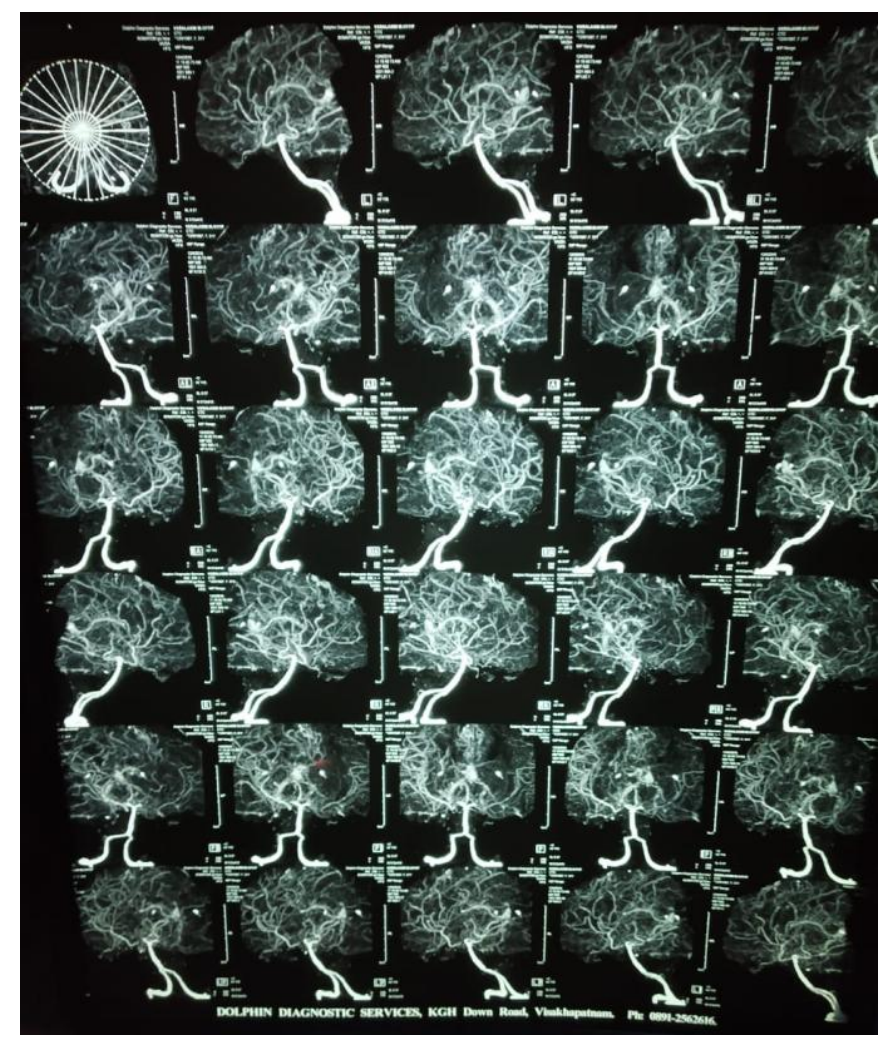

Figure -2: MR Angiography

\section{Treatment}

She was treated conservatively with 20 percent mannitol. Cobalamine, vitamin B-6, folic acid supplementations were given. Physiotherapy of the left upper limb and lower limb was done. The patient was advised for neurosurgery for revascularization procedures. 


\section{Discussion}

Moyamoya vasculopathy is a rare cause of stroke in young. It can cause both ischemic and hemorrhagic stroke. Ischemic stroke is common in children, and hemorrhagic stroke is seen in adults.

Moyamoya vasculopathy is characterized by Occlusion or stenosis of terminal internal carotid arteries and proximal part of anterior cerebral and middle cerebral arteries. As a compensatory mechanism, collaterals develop from basal perforators. These collaterals gives rise to puff of smoke appearance in carotid angiography.

Moyamoya is a Japanese word which means puff of smoke. ${ }^{1}$ Pathology of moyamoyavasculopathy is due to hyperplasia of tunica intima with smooth muscle Proliferation in the vessel wall of the involved arteries. The collaterals have a thin wall with deposits of fibrin and form micro aneurysms. This pathology of moyamoya vasculopathy can be associated with other diseases, or it can present without any associations. The former condition is termed as moyamoya syndrome or secondary moyamoya, and the latter condition is termed as moyamoya disease. $^{2}$

Conditions associated with moyamoya syndrome are shown in table no:2

\section{Table no: 2}

\begin{tabular}{|l|l|}
\hline HEMATOLOGICAl & INFECTION \\
\hline Sickle cell anaemia & Pneumococcal meningitis \\
\hline Essential thrombocythemia & HIV infection \\
\hline Protein C and S deficiency & Hepatitis C infection \\
\hline $\begin{array}{l}\text { Hereditary } \\
\text { Spherocytosis }\end{array}$ & Syphilis \\
\hline Hyperhomocysteinemia & $\begin{array}{l}\text { Tuberculous } \\
\text { meningitis }\end{array}$ \\
\hline AUTOIMMUNE lupus & OTHERS \\
\hline $\begin{array}{l}\text { Systemic } \\
\text { erytematosus }\end{array}$ & Oral contraceptive \\
\hline Sarcoidosis & Head injury \\
\hline Behecets disease & Brain tumor \\
\hline $\begin{array}{l}\text { Antiphospholipid antibody } \\
\text { syndrome }\end{array}$ & \\
\hline
\end{tabular}

\begin{tabular}{|l|}
\hline GENETIC SYNDROMES ASSOCIATED ARE \\
\hline Downs syndrome \\
\hline Turners \\
\hline Neurofibromatosis \\
\hline Marfans syndrome \\
\hline Polycystic kidney disease \\
\hline Tuberous sclerosis \\
\hline
\end{tabular}

Our case has Hyperhomocysteinemia, which is associated with the development of moyamoya syndrome.

There were few case reports of moyamoya syndrome with abnormal thrombophilia profile. There were reports of moyamoya disease with protein $\mathrm{c}$ and protein s deficiency. ${ }^{3}$ A.lentini et al.; described atherosclerotic adult moyamoya disease in a patient with ischemic stroke with Hyperhomocysteinemia. A case of atherosclerotic moyamoya disease presented with ischemic stroke was reported by Cerratoetal. ${ }^{4}$ According to their report, atherosclerosis secondary to increased homocysteine was the likely cause of moyamoya. Hyperhomocysteinemia is identified as an independent risk factor for disease progression. Hyperhomocysteinemia is classified as follows:

Moderate (15 to $30 \mathrm{micromol} / \mathrm{L}$ )

Intermediate (30 to $100 \mathrm{micromol} / \mathrm{L}$ )

Severe (>100 micromol/L)

Hyperhomocysteine damage blood vessels by inducing endothelial dysfunction and creating a prothrombotic state and disordered angiogenesis, which are typical pathological presentations in Moyamoya syndrome.

Hyperhomocysteinemia can be due to both genetic and acquired causes. Moderate hyperhomocysteinemia is mostly due to impaired pathways of remethylation which may be due to deficiency of folate, vitamin b6 and b 12 or deficiency of MTHFR- Methylenetetrahydrofolate reductase enzyme .

In a study by L. Duan, L. Wei, Y. Tian et al., several novel single nucleotide polymorphisms were detected in a gene regulating homocysteine metabolism. Two of the risk alleles are MTHFR and TCN2, which implicates abnormal homocysteine metabolism in Moyamoya syndrome pathogenesis. ${ }^{5}$ Therefore Therapeutic interventions targeting homocysteine may thus benefit some patients with Moyamoya disease.

Management of moyamoya with drugs is not sufficient. Depending on the clinical presentation of the disease, treatment varies. If presented as hemorrhagic stroke, first treat hypertension if 
present. Acute management is conservative treatment aiming at lowering intracranial pressure and managing seizures. There is a better prognosis if presented while symptoms are evolving.

Clinical trials have shown that oral supplementation with the combination of folic acid, B6, and B12vitamins substantially lowers circulating homocysteine levels. ${ }^{6}$

For secondary prevention in case of ischemic stroke different surgical procedures have been used

\section{Conclusion}

In conclusion, patients presenting with recurrent stroke at young age apart from routine CT imaging, we have to consider MRI and MR angiography to look for collaterals formation as in Moyamoya vasculopathy. We need to do thrombophilia profile to look for any associations like hyperhomocysteinemia causing moyamoya syndrome and evaluate for hyperhomocysteinemia causes like vitamin b12 and folate deficiencies.

\section{References}

1. Suzuki J, Takaku A. Cerebrovascular moyamoya disease: disease showing abnormal net-like vessels in base of brain. Archives of neurology. 1969 Mar 1;20(3):288-99.

2. Scott RM, Smith ER. Moyamoya disease and moyamoya syndrome. New England Journal of Medicine. 2009 Mar 19;360(12):1226-37.

3. Çevik B, Acu B, Aksoy D, Kurt S. Protein S deficiency and an adult case with Moyamoya syndrome that presented with primary intraventricular haemorrhage. Balkan medical journal. 2014 Jun 1;2014(2):180-3.

4. Cerrato P, Grasso M, Lentini A, Destefanis E, Bosco G, Caprioli M, Bradac GB, Bergui M. Atherosclerotic adult Moya-Moya disease in a patient with hyperhomocysteinaemia. Neurological Sciences. 2007 Mar 1;28(1):45-7.
5. Duan L, Wei L, Tian Y, Zhang Z, Hu P, Wei Q, Liu S, Zhang J, Wang Y, Li D, Yang W. Novel susceptibility loci for moyamoya disease revealed by a genome-wide association study. Stroke. 2018 Jan;49(1):11-8.

6. Maron, Bradley A, and Joseph Loscalzo. "The treatment of hyperhomocysteinemia." Annual review of medicine vol. $60 \quad$ (2009): 39-54. doi:10.1146/annurev.med.60.041807.123308 1. 